\title{
Extreme Right Axis Deviation
}

National Cancer Institute

\section{Source}

National Cancer Institute. Extreme Right Axis Deviation. NCl Thesaurus. Code C102634.

An electrocardiog raphic finding of a frontal plane QRS axis from -90 to +180 degrees.

(CDISC) 